\title{
EVALUACIÓN DE LA POBLACIÓN DE Athyonidium chilensis Y PARÁMETROS FÍSICO-QUÍMICOS EN PLAYITA BRAVA TACNA
}

\section{EVALUATION OF THE POPULATION OF Athyonidium chilensis AND PHYSICOCHEMICAL PARAMETERS IN PLAYITA BRAVATACNA}

\author{
${ }^{1}$ Freddy Walter Delgado Cabrera \\ ${ }^{2}$ Luis Antonio Espinoza Ramos \\ ${ }^{3}$ Calixto Quispe Pilco
}

\section{RESUMEN}

El presente artículo responde a un proyecto de investigación cuyo objetivo general fue evaluar la población de la holoturia pepino de mar (Athyonidium chilensis) así como medir, de acuerdo a los métodos estándar de la AWWA, los parámetros físico-químicos y biológicos de la zona de Playita Brava del litoral de Tacna.

Se llegó a la conclusión de que existe una dispersión en la población de holoturia pepino de mar (Athyonidium chilensis) en la zona donde se desarrolló el estudio, determinándose que no hay una correlación entre su longitud y su peso (variables dependientes); en consecuencia, se trata de una población variable. Por otro lado, los parámetros físico-químicos están dentro del rango permisible para el desarrollo de holoturia pepino de mar; sin embargo, el factor físico de temperatura presenta una anomalía térmica de hasta $2^{\circ} \mathrm{C}$ según se observó en el muestreo realizado el $19 / 05 / 14$, y de $1{ }^{\circ} \mathrm{C}$ en el muestreo del 04/06/14. Por último, en base a referencias del año 2013 (Informe Envirolab Perú S.A.C.), el fitoplancton presente en Playita Brava está compuesto por divisiones como Bacyllarophyta, Pyrrofhyta, Chrysophita y Euglenophyta, destacando los géneros Lephoylinchus discus (20 cel/l) y Navicula (22 cel/l); en cuanto al zooplancton, destaca el Phyllum Artropoda, dando 5 y 100 organismos/muestra respectivamente en 5 litros.

Palabras Clave: GPS, Pepino de mar, Playita Brava, referenciación geográfica.

\section{ABSTRACT}

The present article responds to a research project whose general objective was to evaluate the population of sea cucumber holothuria (Athyonidium chilensis). As well as measuring, according to the AWWA standard methods, the physical-chemical and biological parameters of the Playita Brava zone of the coastline of Tacna.

It was concluded that there is dispersion in the population of sea cucumber holothuria (Athyonidium chilensis) in the area where the study was developed, it was determined that there is no correlation between its length and its weight (dependent variables); consequently, it is a variable population. On the other hand, the physico-chemical parameters are within the permissible range for the development of sea cucumber holothuria; however, the physical temperature factor has a thermal anomaly of up to $2^{\circ} \mathrm{C}$ as observed in the sampling carried out on $05 / 19 / 14$, and of $1{ }^{\circ} \mathrm{C}$ in the sampling of 04/06/14. Finally, based on references from the year 2013 (Envirolab report Peru S.A.C.), the phytoplankton present in Playita Brava is composed of divisions such as Bacyllarophyta, Pyrrofhyta, Chrysophita and Euglenophyta, highlighting the genera Lephoylinchus discus (20 cel / l) and Navicula (22 cel / 1); and as far as zooplankton, the Phyllum Artropoda stands out, giving 5 and 100 organisms / sample respectively in 5 liters.

Keywords: GPS, Sea cucumber, Playita Brava, geographical referencing..

\footnotetext{
${ }^{1}$ Escuela Profesional de Ingeniería Pesquera. Facultad de Ciencias Agropecuarias. Universidad Nacional Jorge Basadre Grohmann. Tacna- Perú. E-mail: fdelgado2001c@hotmail.com

${ }^{2}$ Escuela Profesional de Ingeniería Pesquera. Facultad de Ciencias Agropecuarias. Universidad Nacional Jorge Basadre Grohmann. Tacna- Perú. E-mail: laer54@hotmail.com

${ }^{3}$ Programa Nacional de Innovación en Pesca y Acuicultura. Lima-Perú. E-mail: calixto_81@hotmail.com
} 


\section{INTRODUCCIÓN}

Los equinodermos son invertebrados que pueden vivir enterrados en la arena, en el fango, entre rocas y arrecifes planos; casi exclusivamente marinos, habitan desde el intermarial hasta las profundidades abisales y son relacionados frecuentemente con algas y corales.

En el Pacífico oriental la pesca de pepino de mar más importante, en la especie Isostichopus fuscus, se realiza en México y en las Islas Galápagos (ToralGranda, 2005); así mismo se viene desarrollando actividades incipientes en Perú y Chile (Guisado, 2005), que se centran en Pattalus mollis y Athyonidium chilensis (Cucumariidae).

Las especies de zonas temperadas, como Cucumaria frondosa en Canadá, generalmente desovan una vez al año en primavera o a inicios del verano (Hamel \& Mercier, 1996). Las holoturias son extremadamente vulnerables a la sobre explotación excesiva debido a su madurez tardía, su reproducción dependiente de la densidad, baja supervivencia de las larvas y la facilidad de captura por los seres humanos.

El notable aumento de los desembarcos y la exportación de holoturias, combinado con la escasez de datos sobre las pesquerías, la pobreza de información biológica y los parámetros de población para las especies comercialmente importantes, así como las pocas medidas de ordenación, son factores que contribuyen a la disminución de las poblaciones de holoturias.

En el presente trabajo se busca conocer las características de la holoturia de mar (Athyonidium chilensis) existente en la zona de Playita Brava, del CP Boca del Río, provincia y región de Tacna; asimismo, determinar los valores de los parámetros físicoquímicos y biológicos de esta zona en donde se desarrolla la especie en estudio.

\section{FUNDAMENTOS TEÓRICOS}

\section{Distribución geográfica}

En Perú, esta especie se distribuye en la bahía de Ancón, playa Las Sombrillas en la Costa Verde, bahía Independencia, bahía San Juan e Ilo; Punta Gaviota en Chile $\left(11^{\circ} 45^{\prime} \mathrm{S}\right.$ y $42^{\circ} 03^{\prime} 50^{\prime \prime} \mathrm{S}$, correspondiente a cada país) (IMARPE, 2013).

\section{Biología y ecología del pepino de mar}

El pepino de mar tiene un cuerpo blanquecino-grisáceo blando y cilíndrico, con pies ambulacrales presentes en la pared del cuerpo. Presenta 5 pares de tentáculos ramificados de color negro-verdoso. En el medio natural solo es posible observar los cinco tentáculos que rodean la boca, estos son bífidos (divididos en dos) $\mathrm{y}$ arborescentes, con numerosas ramificaciones pequeñas (IMARPE, 2013).

La mayoría de los pepinos desovan por difusión liberando esperma y oocitos directamente en la columna de agua. Las hembras pueden liberar miles de millones de oocitos en un solo evento de desove. Algunas especies comerciales lo hacen varias veces al año o periódicamente cada año, tales como Isostichopus fuscus en Ecuador, y Holothuria scabra en las islas Salomón (Hamel et al., 2001). En cuanto a las especies de zonas temperadas, como ya se señaló antes, estas generalmente desovan una vez al año en primavera o a inicios del verano (Hamel \& Mercier, 1996).

Para Conand (1996), el crecimiento en los pepinos de mar ha sido difícil de evaluar por cuanto estos no han respondido bien a los métodos convencionales de marcaje. De igual forma, Shelley (1985) estimó el crecimiento en $14 \mathrm{~g} /$ mes de H. scabra y de 19 a 27 $\mathrm{g} / \mathrm{mes}$ para Actinopyga echinites. Uthicke \& Benzie (1994) encontraron un incremento modesto de peso en Stichopus chloronotus de 70 a $80 \mathrm{~g} / \mathrm{mes}$, y Franklin (1980) mostró que su crecimiento disminuye a medida que el animal se hace más grande.

En función de su capacidad de desplazamiento, los pepinos de mar son lentos y pueden ser considerados "sedentarios" (Reichenbach, 1999).

Las reservas marinas pueden ser relativamente pequeñas, pero las especies que no migran dentro y fuera de estas fácilmente van conformando redes (Sale et al., 2005).

\section{Alimentación}

Se alimentan de detritus, bacteria y diatomeas mezcladas con los sedimentos del fondo marino (Conand \& Byme 2006). Algunas especies se entierran en el sedimento y se cree que ayudan a oxigenar las capas superficiales de los sedimentos, jugando un rol en la bioturbación (Purcell, 2004).

\section{Consumo}

China es el principal país consumidor, los pepinos de mar son también apreciados en los países del sureste de Asia y por asiáticos que viven en el extranjero (Ferdhouse, 2004).

\section{MATERIALES Y MÉTODOS}

Tomando en cuenta la naturaleza de los objetivos planteados, esta investigación responde al tipo descriptivo o investigación aplicada mediante evaluación directa. 


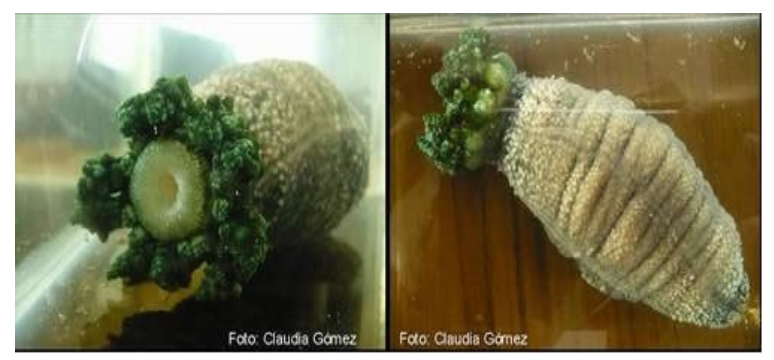

Figura 1: Athyonidium chilensis

Fuente: IMARPE (2013)

\section{Técnicas e instrumentos de recolección de datos}

La aplicación de la unidad de muestreo se basa principalmente en las características de los recursos a evaluar, características operativas del sector, factibilidad técnica y capacidad de la asimilación de la metodología por parte de las organizaciones de pescadores artesanales participantes de la actividad. Inicialmente, se planteó la aplicación de un cuadrante de $1 \mathrm{~m}^{2}$ para la evaluación del recurso principal y de cuadrantes de $0,25 \mathrm{~m}^{2}$ para la evaluación de los recursos secundarios. Robothan (1995).

Finalmente, se establecieron cuadrantes de $120 \mathrm{~m}^{2}$ de superficie.

\section{Métodos y técnicas de procesamiento y análisis de datos}

Para la determinación de la estructura comunitaria, se tuvo en cuenta la distribución y cuantificación directa de las especies, asimismo se determinó los principales indicadores comunitarios. Las áreas fueron divididas en transectos, equidistantes y perpendiculares a la costa, con una separación previamente establecida. En cada transecto se estableció estratos a diferentes niveles de profundidad, determinándose un estrato superior, uno medio y un estrato profundo. Fueron registrados datos de posición geográfica, empleando un GPS map 76S, datos de profundidad y área efectiva o área rocosa disponible (hábitat) en porcentaje (cobertura espacial) y confirmación visual del sustrato.

\section{RESULTADOS}

\section{Referenciación geográfica}

Con la ayuda del GPS, se realizó la georreferenciación de las estaciones de monitoreo (E.M. 01; 02; 03 y 04), tal como se muestra en la Figura 2

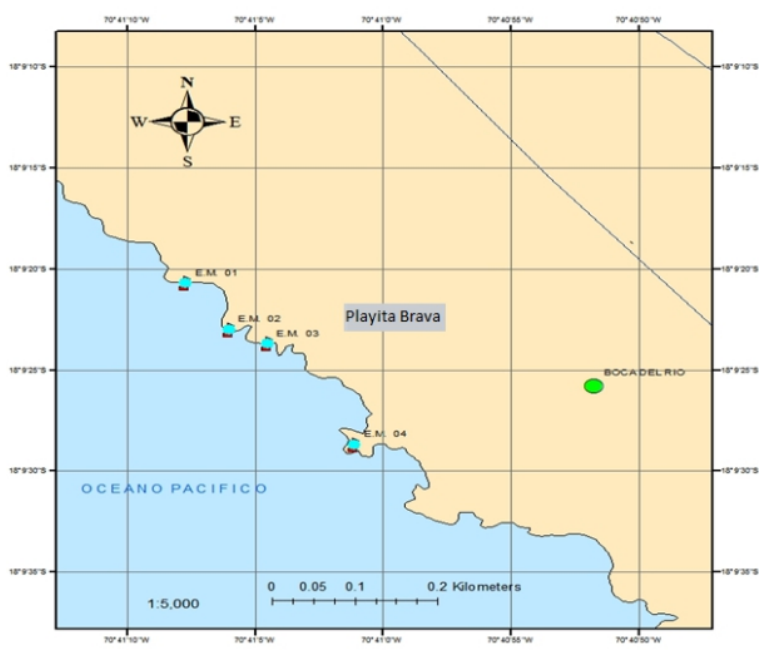

Figura 2. Mapa de ubicación de las estaciones de monitoreo de la zona de trabajo de esta investigación. Playita Brava $29 / 08 / 14$

Ubicación: Playita Brava, Latitud 18 9' 19.05” y Longitud $70^{\circ} 41^{\prime} 10.93 "$

\section{Trabajo de campo primera etapa}

Para cumplir con los objetivos del proyecto de investigación, se salió a campo a fin de extraer muestras del recurso pepino de mar (Athyonidium chilensis). Dichas muestras fueron tomadas en la zona de trabajo del proyecto (Playita Brava), con la ayuda de un buzo artesanal. Posteriormente, las muestras fueron trasladadas en cajas de tecnopor con agua de mar, al laboratorio de acuicultura de la Escuela de Ingeniería Pesquera donde se registraron las medidas de talla, peso y eviscerado.

De un registro de 49 pepinos de mar se obtuvo longitudes totales entre 5,4 y $13,5 \mathrm{~cm}$, con moda en 10 $\mathrm{cm}$, para biomasa 162 gramos; por cuanto se ha determinado una longitud promedio de $9,3 \mathrm{~cm}$ (ver figuras 3 y 4 ).

El grado de correlación fue de - 0,014 , lo cual indica que no existe una relación entre la longitud y peso debido a que los datos se encuentran muy dispersos.

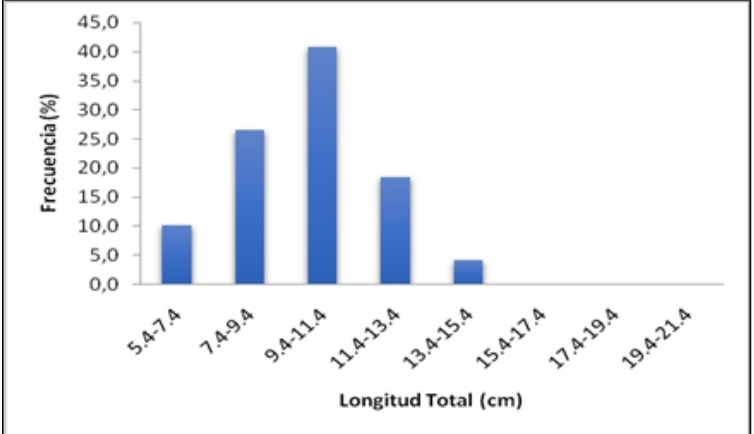




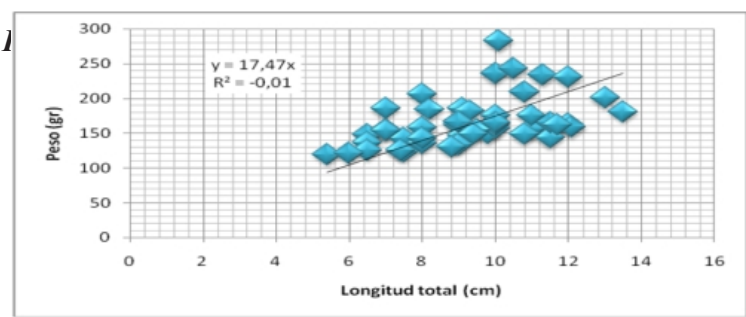

Figura 4. Curva de correlación Longitud - Peso del pepino de mar

Evaluación de los parámetros físico-químicos del agua de mar en la zona de desarrollo del proyecto: Playita Brava. Litoral de Tacna

Dentro del trabajo de campo efectuado, se ha obtenido muestras de agua de mar en 4 oportunidades: el $19 / 05 / 2014$; el $04 / 06 / 2014$; el $18 / 07 / 2014$; y el 20/08/2014. Los análisis correspondientes a cada una de las muestras fueron realizados en el laboratorio de Tecnología Pesquera.

Los resultados de los parámetros físico-químicos obtenidos indican que están dentro del rango permisible para el desarrollo del pepino de mar. No obstante, es importante destacar que la temperatura del agua en el mes de mayo tuvo una anomalía térmica de $1{ }^{\circ} \mathrm{C}$, pero se ha regularizado en los meses de junio y julio. Esto concuerda con los informes de las instituciones encargadas de hacer estos registros, quienes informaron que el presente año se presentaría el fenómeno de El Niño de moderado a fuerte, situación que a la fecha ya se ha normalizado (ver Tabla 1).

Tabla 1. Parámetros físico-químicos del agua de mar en la zona del proyecto

\begin{tabular}{|c|c|c|c|c|}
\hline & $19 / 05 / 2014$ & $04 / 06 / 2014$ & $18 / 07 / 2014$ & $20 / 08 / 2014$ \\
\hline Temperatura & 17,50 & 16,50 & 15,40 & 15,50 \\
\hline $\mathrm{pH}$ & 7,71 & 7,64 & 7,74 & 7,62 \\
\hline $\begin{array}{l}\text { Conductividad } \\
\text { eléctrica } \\
\mathrm{mS} / \mathrm{cm}\end{array}$ & 55,20 & 54,20 & 53,94 & 53,80 \\
\hline $\begin{array}{l}\text { Carbonatos } \\
\mathrm{mg} / \mathrm{l}\end{array}$ & 25,80 & 25,80 & 25,20 & 25,60 \\
\hline $\begin{array}{l}\text { Bicarbonatos } \\
\mathrm{mg} / \mathrm{l}\end{array}$ & 111,50 & 109,83 & 120,20 & 109,80 \\
\hline $\begin{array}{l}\text { Cloruros } \\
\mathrm{mg} / 1\end{array}$ & 19698,24 & 18600,22 & 19663,09 & 19650,08 \\
\hline Dureza mg/l & 6386,00 & 6719,77 & 6957,63 & 6952,61 \\
\hline $\begin{array}{l}\text { Alcalinidad } \\
\mathrm{mg} / 1\end{array}$ & 134,00 & 133,00 & 115,20 & 120,30 \\
\hline $\mathrm{CO} 2 \mathrm{mg} / \mathrm{l}$ & 0,00 & 0,00 & 0,00 & 0,00 \\
\hline $\begin{array}{l}\text { Salinidad } \\
00 / 0\end{array}$ & 35,88 & 35,23 & 35,06 & 35,33 \\
\hline
\end{tabular}

\section{Trabajo de campo segunda etapa}

Durante el desarrollo del proyecto se registró 49 pepinos de mar en los cuales se obtuvo longitudes totales entre 5,6 y $14,4 \mathrm{~cm}$, con moda en $10 \mathrm{~cm}$, para biomasa 154 gramos; el promedio de la longitud quedó determinado en $8,6 \mathrm{~cm}$. El grado de correlación fue de 0,043 ; lo que indica que no existe una relación entre la longitud y peso debido a que los datos se encuentran muy dispersos (ver Figura 5).

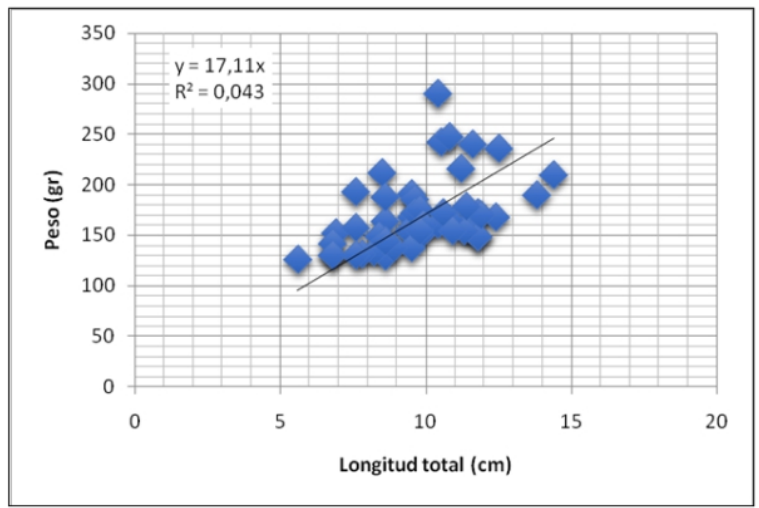

Figura 5. Curva de correlación Longitud -Peso del pepino de mar

Evaluación de los parámetros físico-químicos del agua de mar en la zona de desarrollo del proyecto: Playita Brava. Litoral de Tacna

Durante el trabajo de campo realizado, se obtuvo muestras de agua de mar para su análisis correspondiente en 4 oportunidades. Estas salidas se efectuaron en las siguientes fechas: 03/09/2014; $24 / 09 / 2014 ; 10 / 10 / 2014 ; 15 / 10 / 2014$. Igual que en la etapa anterior, los análisis también se hicieron en el laboratorio de Tecnología Pesquera.

Los resultados de los parámetros físico-químicos obtenidos indican que están dentro del rango permisible para el desarrollo del pepino de mar, antes bien, es importante señalar que la temperatura del agua en los meses de octubre y noviembre tuvo una anomalía térmica de $1,5^{\circ} \mathrm{C}$. Dicha situación ya se había anunciado en los informes oceanográficos del IMARPE (Instituto del Mar del Perú); asimismo se debe señalar que este valor de la temperatura corresponde a la zona superficial del mar, la cual comprende además la orilla donde habita el recurso en estudio. Queda ratificada, de este modo, la presencia del fenómeno de El Niño, entre leve y moderado.

Respecto a los valores del $\mathrm{pH}$, podemos indicar que el valor mínimo - 7,60 - se registra en el mes de setiembre, y el valor máximo - 7,70 - durante el mes de octubre.

En cuanto a la salinidad, su valor mínimo fue de 35,02 y el máximo de 35,68: valores que están dentro del rango normal.

Así mismo los demás parámetros presentan valores dentro de lo normal, lo cual permite el desarrollo de las especies en el litoral, específicamente, en la zona de desarrollo del proyecto (ver Tabla 2). 
Tabla 2. Parámetros físico-químicos del agua de mar en la zona del proyecto

\begin{tabular}{|c|c|c|c|c|}
\hline & $03 / 09 / 2014$ & $24 / 09 / 2014$ & $10 / 10 / 2014$ & $15 / 10 / 2014$ \\
\hline $\begin{array}{l}\text { Temperatura } \\
{ }^{\circ} \mathrm{C}\end{array}$ & 18,20 & 18,50 & 19,00 & 18,80 \\
\hline $\mathrm{pH}$ & 7,61 & 7,60 & 7,70 & 7,63 \\
\hline $\begin{array}{l}\text { Conductividad } \\
\text { eléctrica } \\
\mathrm{mS} / \mathrm{cm}\end{array}$ & 55,30 & 54,00 & 53,90 & 53,80 \\
\hline $\begin{array}{l}\text { Carbonatos } \\
\mathrm{mg} / \mathrm{l}\end{array}$ & 25,70 & 25,750 & 25,30 & 25,50 \\
\hline $\begin{array}{l}\text { Bicarbonatos } \\
\mathrm{mg} / \mathrm{l}\end{array}$ & 110,50 & 109,80 & 120,30 & 109,70 \\
\hline $\begin{array}{l}\text { Cloruros } \\
\mathrm{mg} / \mathrm{l}\end{array}$ & 19698,00 & 18610,20 & 19659,09 & 19652,09 \\
\hline Dureza mg/l & 6384,00 & 6716,77 & 6952,63 & 6950,61 \\
\hline $\begin{array}{l}\text { Alcalinidad } \\
\mathrm{mg} / \mathrm{l}\end{array}$ & 133,00 & 134,00 & 116,20 & 121,32 \\
\hline $\begin{array}{l}\mathrm{CO} 2 \mathrm{mg} / 1 \\
\text { Salinidad } \\
00 / 0\end{array}$ & $\begin{array}{r}0,00 \\
35,68\end{array}$ & $\begin{array}{r}0,00 \\
35,20\end{array}$ & $\begin{array}{r}0,00 \\
35,02\end{array}$ & $\begin{array}{r}0,00 \\
35,30\end{array}$ \\
\hline
\end{tabular}

De la figura 6 a la 13, se muestra el comportamiento de temperatura, $\mathrm{pH}$, conductividad eléctrica, cloruros, dureza, salinidad, carbonatos, bicarbonatos, durante el periodo de trabajo.

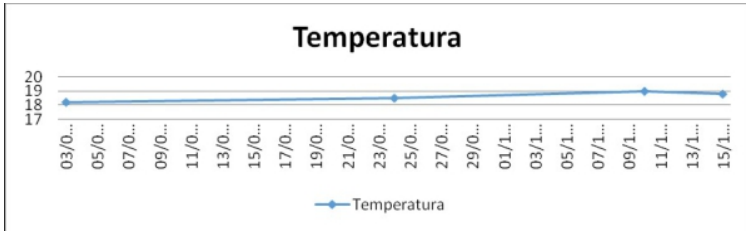

Figura 6. Temperatura del agua de mar

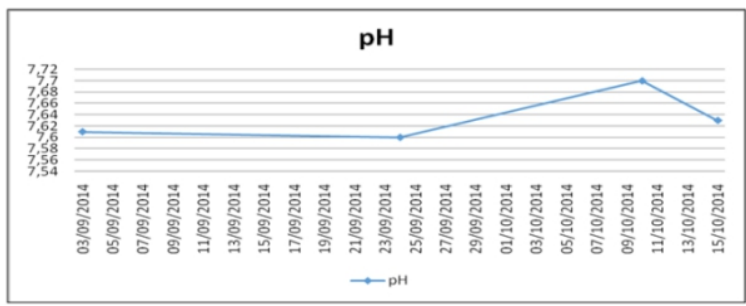

Figura 7. Determinación del pH del agua de mar

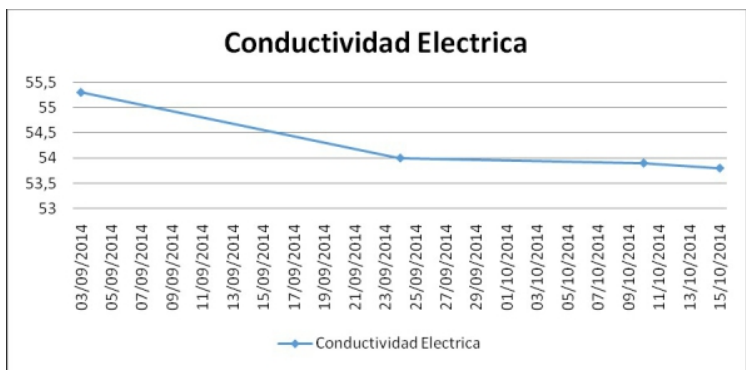

Figura 8. Determinación de la conductividad térmica del agua de mar

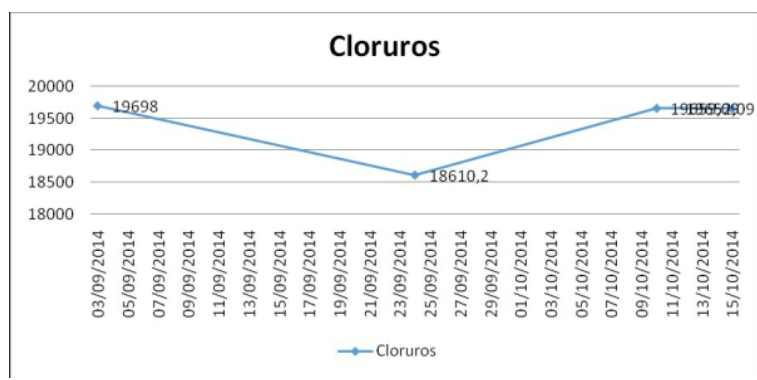

Figura 9. Determinación de cloruros del agua de mar

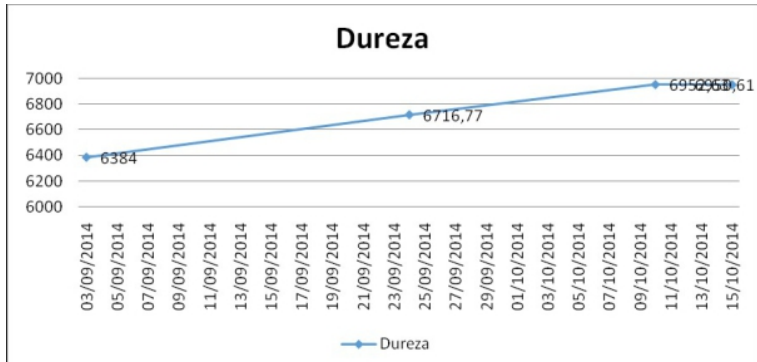

Figura 10. Determinación de la dureza del agua de mar

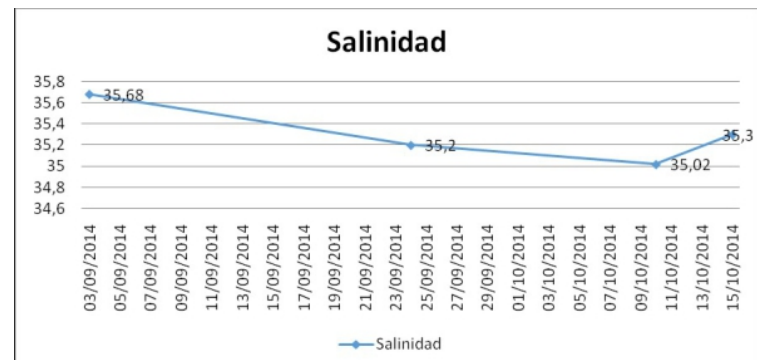

Figura 11. Determinación de salinidad del agua de mar

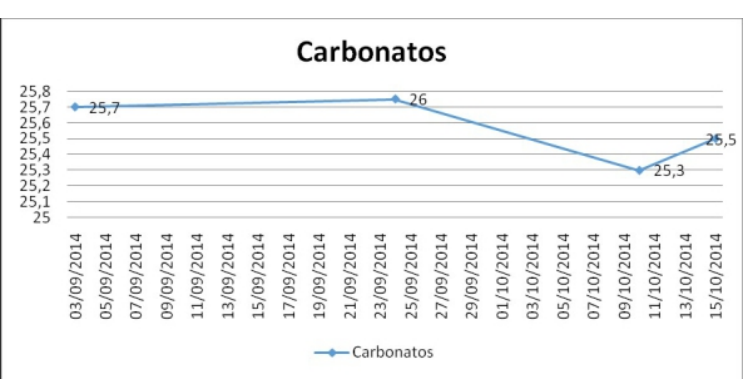

Figura 12. Determinación de carbonatos del agua de mar

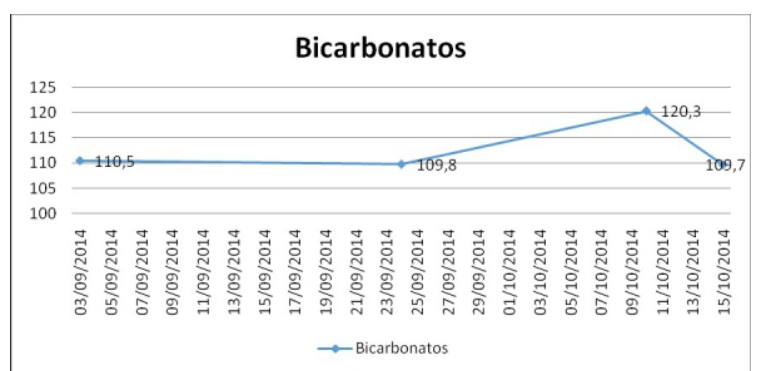

Figura 13. Determinación de Bicarbonatos en el agua de mar

\section{CONCLUSIONES}

Existe una dispersión en la población de holoturia pepino de mar (Athyonidium chilensis) en la zona de 
Playita Brava del litoral de Tacna, puesto que no hay una correlación entre la longitud y el peso (variables dependientes); en consecuencia, la especie objeto de estudio que se halla en la zona de trabajo corresponde a una población variable. Esta población se encuentra en una zona no profunda, principalmente constituida de conchuela y arena; lo cual la convierte en un área protegida de las mareas, haciendo fácil su captura.

Los parámetros físico-químicos están dentro del rango permisible para el desarrollo de holoturia pepino de mar; aunque el factor de temperatura presentó una anomalía térmica de hasta $2^{\circ} \mathrm{C}$, según se observó en el muestreo realizado el $19 / 05 / 14$; y de $1{ }^{\circ} \mathrm{C}$, en el muestreo realizado el 04/06/14.

Por referencias del año 2013 (Informe Envirolab Perú S.A.C.), se sabe que el fitoplancton presente en la zona de Playita Brava está compuesto por divisiones como Bacyllarophyta, Pyrrofhyta, Chrysophita y Euglenophyta, donde destacan los géneros Lephoylinchusdiscus (20 cel/l) y Navicula (22 cel/l). En cuanto al zooplancton destaca el Phyllum Artropoda, dando 5 y 100 organismos/muestra en cada 5 litros.

\section{RECOMENDACIONES}

A fin de determinar el efecto del factor físico de temperatura, es importante desarrollar un programa de investigación conjunta con el Instituto del Mar del Perú, debido a que este factor estaría siendo alterado por el cambio climático.

Se recomienda compartir experiencias de planes de manejo y ordenamiento del recurso con los pescadores artesanales del litoral, especialmente con los de Boca del Río, para mejorar las condiciones de estudio.

\section{REFERENCIAS BIBLIOGRÁFICAS}

Conand, C. \& Byrne, M. (2006). "A review of recent developments in the world of sea cucumber fisheries".

Conand, C. (1996). "Asexual reproduction by fission in Holothuria atra": variability of some parameters in populations from the tropical Indo Pacific.

Ferdhouse, F., (2004). "World markets and trade flows of sea cucumber/beche-de-mer". In: Lovatelli A., Conand C., Purcell S., Paper. No. 463. FAO, Roma. pp. 101-117.

Franklin, S.E. 1980. "The reproductive biology and some aspects of the population ecology of the holothurians Holothuria leucospilota (Brandt) and Stichopus chloronotus
(Brandt)". University of Sydney, Sydney.

Guisado, C. (2005). CONICYT Project: "Development of the cultivation technology of the sea cucumber Athyonidium chilensis, in the Chilean South Central Region”. SPC Bechede-mer Information Bulletin 21, p. 34

Hamel, J.-F. et al. (2001). "The sea cucumber Holothuria scabra (Holothuroidea: Echinodermata)": its biology and exploitation as beche-de-mer. Advances in Marine Biology, 41, pp. 129-233.

Hamel, J.-F. \& Mercier A. (1996). Early development, settlement, growth and spatial distribution of the sea cucumber Cucumaria frondosa (Echinodermata: Holothuroidea). Canadian Journal of Fisheries and Aquatic Sciences, 53, pp. 253-271.

INSTITUTO DEL MAR DEL PERÚ (2013). "Laboratorio Costero de Ilo". "Estudio Base del Banco Natural de Pocoma". Área de investigación y manejo de recursos bentónicos.

Purcell, S.W. (2004). "Rapid growth and bioturbation activity of the sea cucumber Holothuria scabra in earthen ponds". Proceedings of Australasian Aquaculture 2004, p. 244.

Reichenbach, N. (1999). "Ecology and fishery biology of Holothuria fuscogilva (Echinodermata: Holothuroidea) in the Maldives", Indian ocean. Bulletin of Marine Science, 64, pp. $103-113$

Robothan, H. (1995). "Curso Regional”; El muestreo en poblaciones biológicas. Programas de Cooperación Técnica para la Pesca CEEVECEPALA.

Sale, P. et al. (2005). "Critical science gaps impede use of no-take fishery reserves". Trends in Ecology and Evolution, 20, pp. 74-80.

Shelley, C. (1985). "Growth of Actinopyga echinites and Holothuria scabra (Holothurioidea: Echinodermata) in Papua New Guinea". In: C. Gabri'e et al. (eds). Ecole Pratique des Hautes Etudes, Moorea, French Polynesia. pp. 297-330.

Toral-Granda, V. (2005) "Islas Galápagos Sea cucumber fisheries": global analysis of stocks, management measures and drivers of overfishing Fish and fisheries 14 (1), pp. 3459.

Uthicke, S. \& Benzie, J. (2001). "Effect of loche de mer fishing on densities and size structure of Holothuria nobilis (Echinodermata: Holothuridae)" populations on the Great Barrier Reef. Coral reefs. 